\title{
Assessing the Impact of Aggregate Type on Air Lime Mortar Properties Using Scanning Electron Microscopy
}

\author{
Sarah Scannell, Mike Lawrence and Pete Walker \\ Building Research Establishment Centre for Innovative Construction Materials, Department of Architecture and Civil Engineering, \\ University of Bath, Bath BA2 7AY, UK
}

\begin{abstract}
In recent years, the need for low energy materials has become increasingly important. With government targets aiming to reduce carbon emissions by $80 \%$ by 2050 , and the construction industry being responsible for $50 \%$ of the UK's carbon emissions, it is of vital importance that positive changes are made. One of these changes is to reduce the carbon footprint of the materials used in construction. Lime mortar has been used for centuries, but since the arrival of cement, its use in modern construction has diminished, in part due to having lower compressive strengths than cement mortar. Air lime mortar, in particular, can be categorised as low energy due to the reabsorption of a significant amount of $\mathrm{CO}_{2}$ during the setting process: carbonation. The current study focuses on the impact of different types of aggregate (limestone and silicate) on air lime mortar strength. Previous research has found that higher strengths can be achieved with the use of limestone aggregate, but little is known about the reasons why. The research presented here looks at a microstructural analysis through use of SEM (scanning electron microscopy) in order to determine reasons behind the strength differences. At early stages of curing, there are clear differences at the interface of binder and aggregate.
\end{abstract}

Key words: Air lime, limestone, silicate aggregate, scanning electron microscopy, carbonation.

\section{Introduction}

\subsection{Background}

Lime mortar has been used for centuries in masonry construction, and the past few decades have seen an increase in restoration of old buildings, where the compatibility of old and new materials is particularly important. Use of cement mortar is inappropriate for a number of reasons. The high compressive strengths reduce the amount of movement that can occur in the structure and consequently, a build-up of stresses would cause the masonry to fail [1]. Additionally, soluble salts can leech out of the cement, causing damaging salt crystallization.

Lime mortars are inherently weak, and research has been shown that higher strengths can be obtained with use of limestone aggregate over silicate aggregate [2]. Since low strengths are synonymous with poor durability, higher strengths could lead to longer

Corresponding author: Sarah Scannell, Ph.D., research fields: limestone aggregate and non-hydraulic lime. lasting mortars. The obtained higher strengths are still much lower than cement mortar strengths so would not have a detrimental effect on existing masonry. Aggregates are primarily used to provide structure to a mortar [3] and their role in mortar strength has been largely underestimated. Despite various studies concluding that limestone aggregates can produce higher strength air lime mortars [2, 4, 5], little is known about the reasons why.

In the current climate, there is a huge demand for the reduction of carbon emissions; Government targets aim to reduce emissions in the UK by $80 \%$ by 2050. The construction industry is responsible for around $50 \%$ of the UK's carbon emissions [6], therefore, it has a responsibility to work towards a reduction. The use of low energy materials, such as the air lime mortar used in this study, can make a significant contribution. Air lime is a low energy binder due to the fact that during the curing process, almost all of the $\mathrm{CO}_{2}$ that was emitted during the manufacturing process is reabsorbed during 
carbonation [7].

The research originated due to lack of knowledge surrounding the effect of aggregate type on mortar properties, particularly compressive strength. Consequently, three limestone aggregates were compared against a silicate sand-CEN (Comité Européen de Normalisation) standard sand to determine firstly the compressive strengths, and secondly whether there were any differences at a microstructural level for the different mixes. SEM (scanning electron microscopy) was used for this analysis.

\subsection{The Role of Carbonation}

Carbonation is the primary chemical reaction that occurs during the setting of air lime mortar, and it is influenced by several factors: water content of a sample, relative humidity of curing, temperature and the porosity of the material.

The carbonation process describes the evolution of a mortar through chemical hardening, and it can be split into two stages. In the first stage, $\mathrm{CO}_{2}$ diffuses into the pores of the mortar reacts with moisture on the surface of the pores or in the air to form carbonic acid. This can be summarized in the equation below:

$$
\mathrm{H}_{2} \mathrm{O}+\mathrm{CO}_{2} \rightarrow \mathrm{H}_{2} \mathrm{CO}_{3}
$$

Following this, the carbonic acid reacts with the portlandite $\left(\mathrm{Ca}(\mathrm{OH})_{2}\right)$, transforming it into calcite $\left(\mathrm{CaCO}_{3}\right)$. Eq. (2) summarizes this:

$$
\mathrm{H}_{2} \mathrm{CO}_{3}+\mathrm{Ca}(\mathrm{OH})_{2} \rightarrow \mathrm{CaCO}_{3}+2 \mathrm{H}_{2} \mathrm{O}
$$

During this process, samples see a weight gain upon transformation of calcium hydroxide to calcium carbonate. Furthermore, the pore structure changes over the carbonation period as a result of the chemical changes; A decrease in total pore volume can be seen [8].

Fick's first law of diffusion can be used to describe the process of $\mathrm{CO}_{2}$ penetrating the pores in mortar:

$$
J=-D(\delta \phi / \delta x)
$$

where, $J=$ diffusion flux, $D=$ diffusion coefficient, $\varphi=$ concentration of substance per unit volume and $x=$ position in length.

The carbonation of lime mortar samples can be measured and assessed in a variety of ways, including but not limited to: phenolphthalein staining; thermogravimetric analysis; scanning electron microscopy; X-ray diffraction; elemental analysis.

\section{Impact of Aggregate Type}

\subsection{Compressive Strength}

Aggregate is largely considered to be an inert filler material, but previous research has shown that the use of different aggregate types in air lime mortar can have an impact on the compressive strength of the mortar $[2,4,5]$. The use of limestone aggregate can yield strengths up to three times higher than mortar made with silicate aggregate [2].

\subsection{Carbonation}

The use of different aggregate types in mortar has an impact on the carbonation of samples. Limestone aggregate in mortars leads to a greater depth of carbonation over the same curing period as silicate aggregate mortars. This is likely to be due to the higher porosity of limestone over silicate aggregate, which in turn leads to a higher overall porosity of the entire sample; $\mathrm{CO}_{2}$ can penetrate the sample much more readily. Skoulikidis et al. [9] looked at the properties of hydrated lime in the use of consolidation of the surface of stones in monuments, and it found that when $6 \%$ calcite was added to the calcium hydroxide, the rate of carbonation increased. It was suggested that $\mathrm{Ca}(\mathrm{CO})_{3}$ is a seed for crystallization, agreeing that a deeper carbonation can consequently occur.

\section{Materials and Methods}

Air lime mortar specimens (using calcium lime CL90 hydrated lime) of dimensions $40 \mathrm{~mm} \times 40 \mathrm{~mm} \times 160 \mathrm{~mm}$ were manufactured in accordance with BS EN 1015-11: 1999 [10], in order for compressive strength testing to be undertaken. Samples were cured in air and tested for flexural and 
compressive strength at 28 days. Three of the mixes were made with different limestone aggregates (Portland, Ham Hill and Stoke Ground Bath Stone), while the fourth was made with a silicate sand. A binder/aggregate $(B / A g)$ ratio of $1: 3$ by volume was used for all mixes. In order for the tests to be able to isolate differences in mortar properties based on aggregate type, the grain size distribution of all limestone aggregates were made to match that of the silicate sand (standard sand CEN 196-1) for one set of mixes. The other set used the limestone aggregates "as supplied" from the quarry but with no particles larger than $4 \mathrm{~mm}$.

An additional 2 4-mm fraction was added to all five aggregates in order to help reducing shrinkage in the mortar specimens. Table 1 shows the sieve sizes used for the "standard" grading, while Table 2 shows the "as supplied" grading of the limestone aggregates. The particle size distribution can be seen in Fig. 1.

Due to the importance of workability of a mortar, it was decided that rather than having a constant water/binder $(w / b)$ ratio, the flow would be kept constant

Table 1 Sieve sizes for "standard" grading.

\begin{tabular}{ll}
\hline Sieve size $(\mathrm{mm})$ & Percentage of passing $(\%)$ \\
\hline 4 & 100 \\
2 & 88 \\
1.6 & 81.84 \\
1 & 58.96 \\
0.5 & 29.04 \\
0.16 & 11.44 \\
0.08 & 0 \\
\hline
\end{tabular}

Table 2 Sieve sizes for "as supplied" grading.

\begin{tabular}{llll}
\hline Sieve size $(\mathrm{mm})$ & \multicolumn{3}{c}{ Percentage of passing $(\%)$} \\
\cline { 2 - 4 } & Ham Hill & Portland & Stoke Ground \\
\hline 4 & 90.10 & 99.70 & 92.90 \\
2 & 75.30 & 97.40 & 78.80 \\
1 & 59.40 & 87.70 & 61.70 \\
0.5 & 55.30 & 68.80 & 43.40 \\
0.25 & 42.30 & 30.30 & 25.70 \\
0.125 & 31.40 & 13.40 & 14.10 \\
0.063 & 15.20 & 3.5 & 4.30 \\
\hline
\end{tabular}

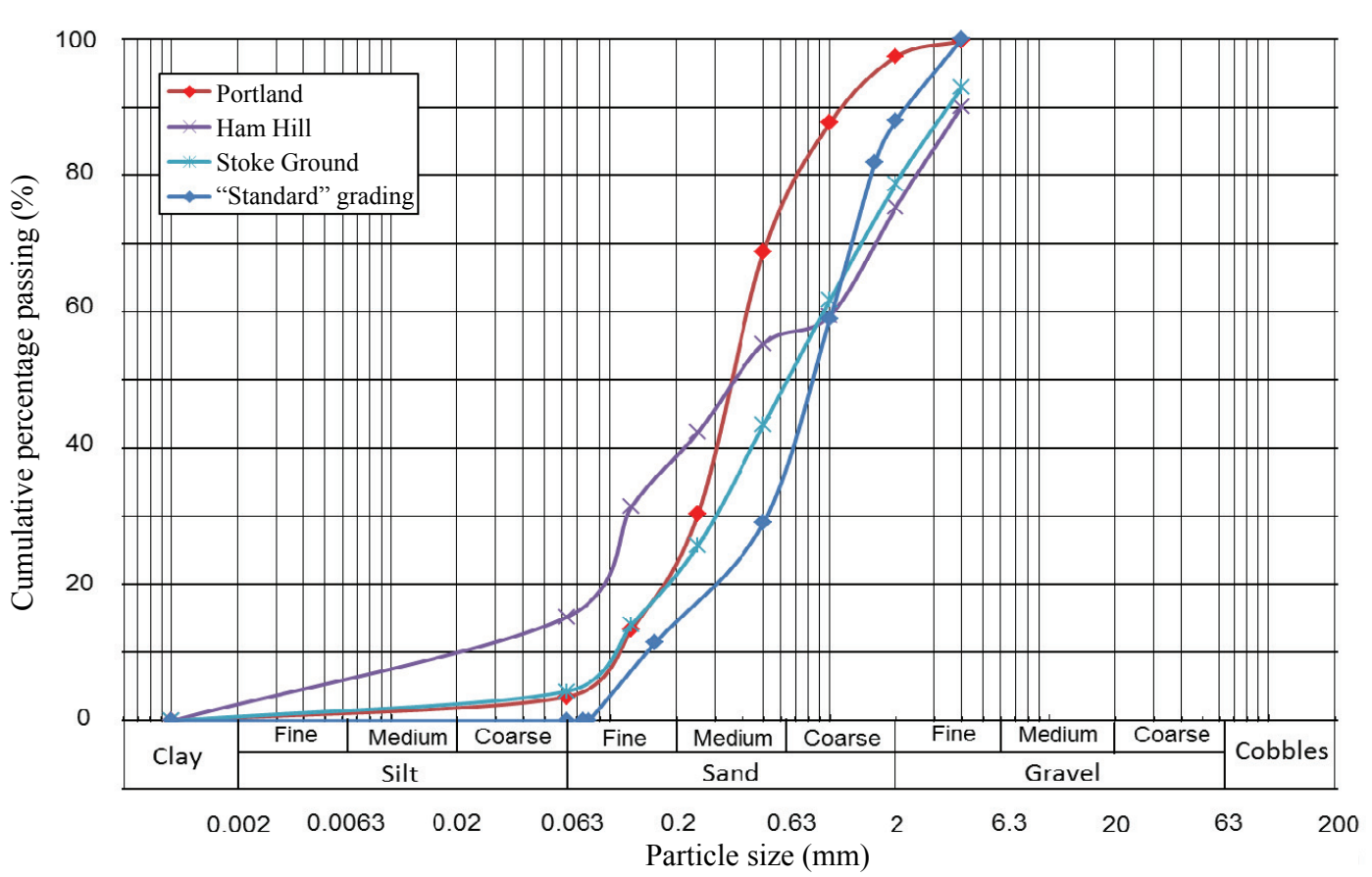

Fig. 1 Particle size distribution of all aggregates. 
at $14 \mathrm{~cm} \pm 0.5 \mathrm{~cm}$. The rationale for this approach was that, since the compressive strength of air lime mortars is insensitive to the $w / b$ ratio within a normal range of workability, a flow representative of that which a mason would use would be more representative. As can be seen from Table 3, significantly more water is required for the stone aggregate mortars due to the greater porosity of the aggregate, which competes with the lime for the available water.

The flow table test measures consistency of a mortar. Mortar is tamped down into a truncated cone, then once the cone is removed, 15 drops of the table are made at a rate of $1 \mathrm{~s}^{-1}$. The diameter of the mortar spread is then measured. This was done in accordance with BS EN 1015-3: 1999 [11].

Tables 3 and 4 show the mix specifications used for each of the mixes in order to achieve a flow of $14 \mathrm{~cm} \pm 0.5 \mathrm{~cm}$.

The $w / b$ ratio is known to impact strength of cement mortars and hydraulic lime mortars. Abrams' Law shows the relationship between the strength of fully compacted concrete and the $w / b$ ratio (Eq. (4)):

$$
f_{c}=K_{1} / K_{2}{ }^{w / c}
$$

where, $f_{c}$ is the water/cement ratio, $w / c$ is the water/cement ratio by volume, $K_{1}$ and $K_{2}$ are constants. Although compressive strength in cementitious mortars is known to follow Abram's Law, being inversely proportional to water/cement ratio $[12,13]$ have shown that for air lime mortars, with the exception of the lowest water/lime ratio, there is very little difference in the compressive strengths of the mortars with increasing water content.

\section{Experimental Results}

\subsection{Compressive Strength}

Results from the compressive strength testing confirmed the findings in the literature that limestone aggregates can produce higher strength mortars than silicate aggregates. Fig. 2 shows the average compressive strengths of the samples at 28 days curing at $55 \% \mathrm{RH}$ (relative humidity), where it is clear to see that the standard sand mortar was the weakest in compression.

While there is a possibility that the higher strengths are in part due to the difference in angularity of the aggregates (the limestone aggregates are more angular), SEM analysis suggests that something more complex is occurring.

\subsection{SEM (Scanning Electron Microscopy)}

Samples were taken from the outer edge of each sample immediately after compressive strength testing, and placed in glass jars filled with nitrogen so as to prevent further carbonation occurring, thus effectively freezing the samples at 28 days curing. The used microscope was JEOL SEM6480LV. It was used under low vacuum conditions.

Fig. 3 shows a Ham Hill aggregate mortar after

Table 3 Mix design for 1:3 "standard" samples.

\begin{tabular}{lllll}
\hline Sample & Lime $(\mathrm{g})$ & Aggregate $(\mathrm{g})$ & Water $(\mathrm{g})$ & $w / b$ \\
\hline Ham Hill & 150 & 2,243 & 458 & 3.05 \\
Portland & 150 & 2,687 & 455 & 3.03 \\
Stoke Ground & 150 & 2,202 & 351 & 2.34 \\
Standard sand & 150 & 1,571 & 150 & 1.00 \\
\hline
\end{tabular}

Table 4 Mix design for 1:3 "as supplied" samples.

\begin{tabular}{lllll}
\hline Sample & Lime $(\mathrm{g})$ & Aggregate $(\mathrm{g})$ & Water $(\mathrm{g})$ & $w / b$ \\
\hline Ham Hill & 150 & 2,243 & 579 & 3.86 \\
\hline Portland & 150 & 2,687 & 542 & 3.61 \\
Stoke Ground & 150 & 2,202 & 390 & 2.60 \\
\hline
\end{tabular}




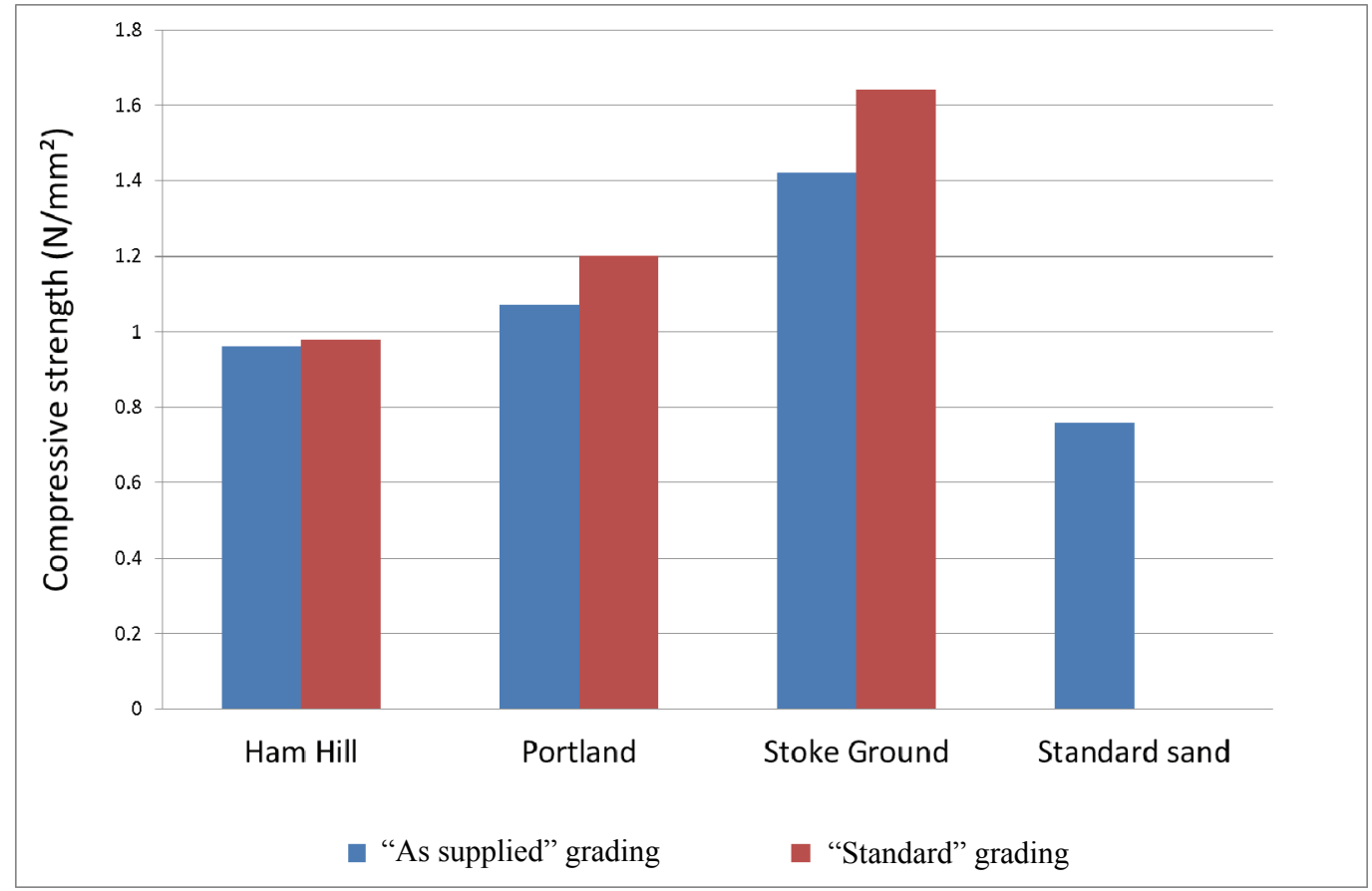

Fig. 2 Average compressive strength at 28 and 360 days.

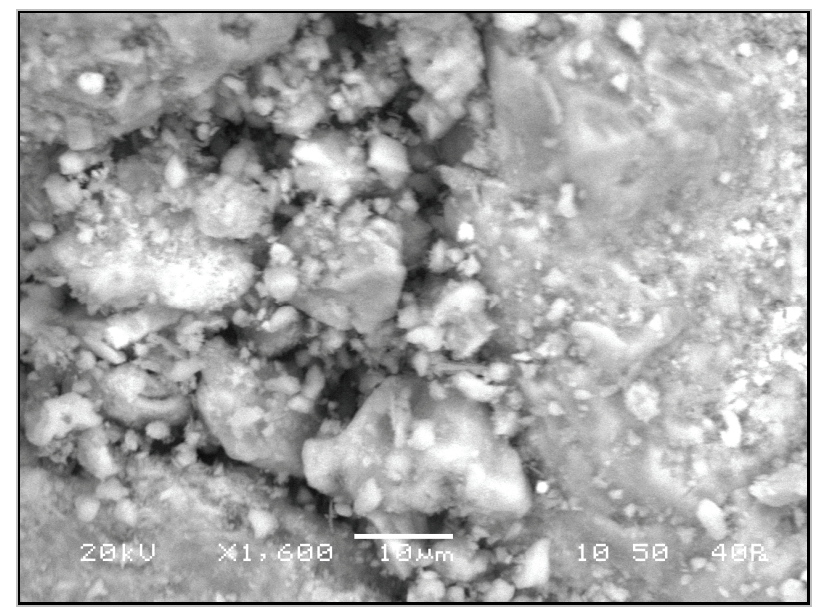

Fig. 3 Ham Hill mortar "standard" grading.

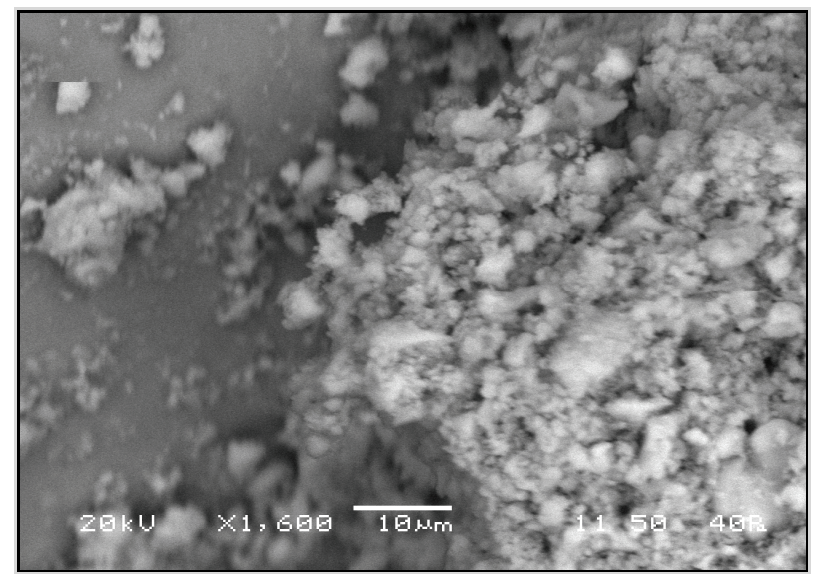

Fig. 4 Standard sand mortar.
28 days curing at $1,600 \times$ magnification. The right-hand third of the image is a section of aggregate, which has some calcite crystals on the surface. To the left of the image, calcite crystals can be seen adhering to the aggregate. Similarly, Fig. 4 shows the standard sand aggregate mortar at the same magnification.

The calcite crystals that are on the surface of the aggregate appear to be in larger clusters than in Fig. 3. Moreover, the aggregate that can be seen in Fig. 4 has a smoother surface than the Ham Hill in Fig. 3.

Despite having the highest compressive strength, the sample containing Stoke Ground aggregate with the "standard" grading contained more micro-cracking than the other samples, which can be seen in Fig. 5, which was taken at $800 \times$ magnification. The crack measures around $75 \mu \mathrm{m}$ in length, and appears to be at the binder/aggregate interface.

It is clear from the image of the Portland aggregate mortar with "standard" grading (Fig. 6) that some portlandite still exists in the sample. This can be seen in the center of the bottom of the image. Furthermore, calcite crystals appear small and are continuous over the sample.

In the Ham Hill "as supplied" sample in Fig. 7, 
some portlandite still exists, with crystals of around $7 \mu \mathrm{m}$.

Cracking was also found with the "as supplied" Stoke Ground samples, seen in Fig. 8, although many of these are around $250 \mu \mathrm{m}$, much larger than in the

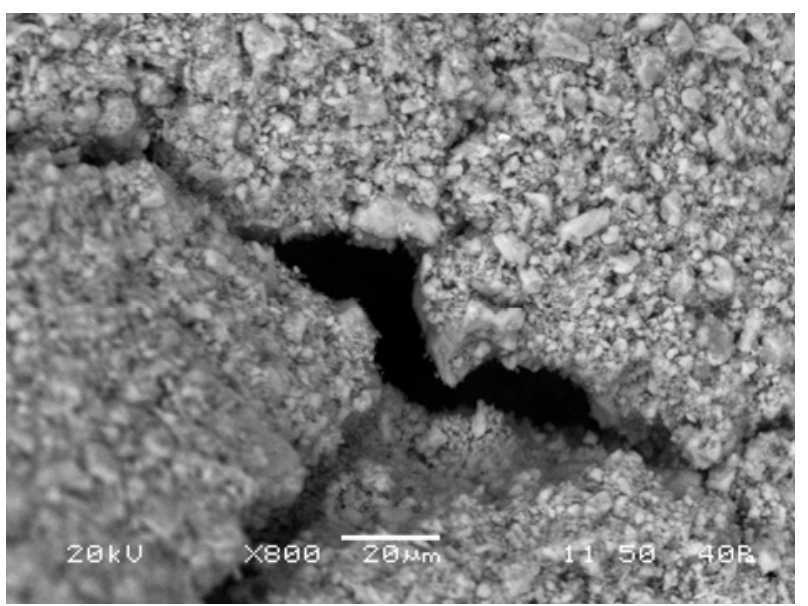

Fig. 5 Stoke Ground mortar "standard” grading.

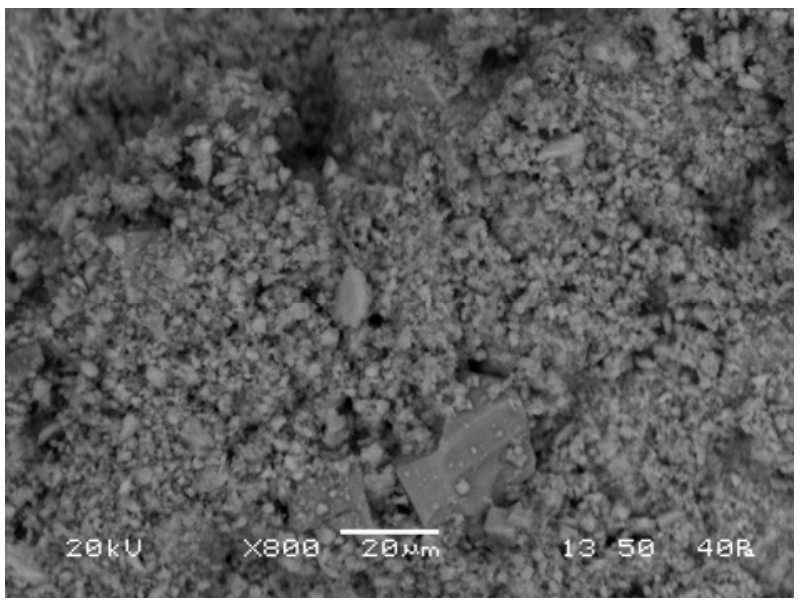

Fig. 6 Portland mortar "standard" grading.

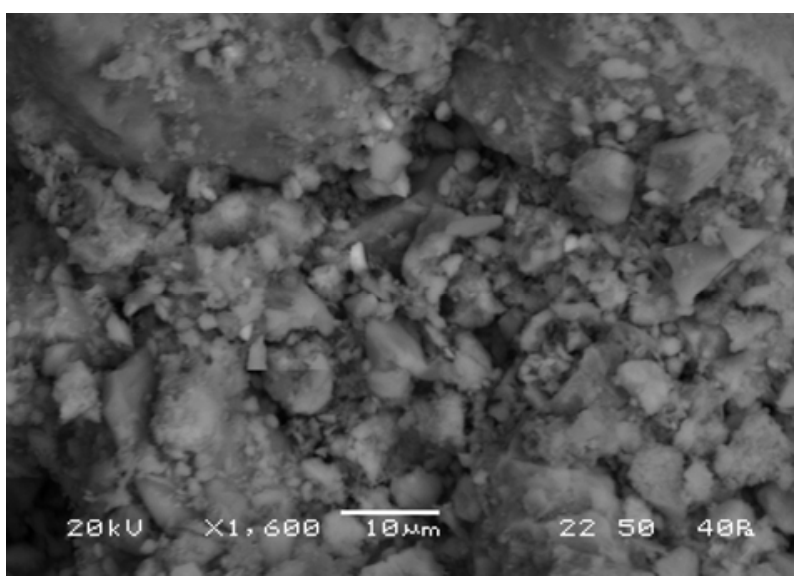

Fig. 7 Ham Hill mortar “as supplied” grading.

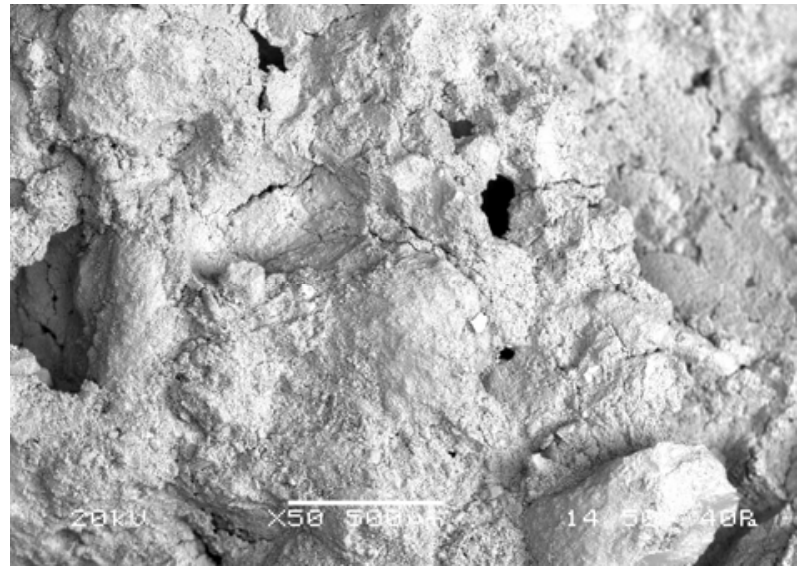

Fig. 8 Stoke Ground mortar “as supplied” grading.

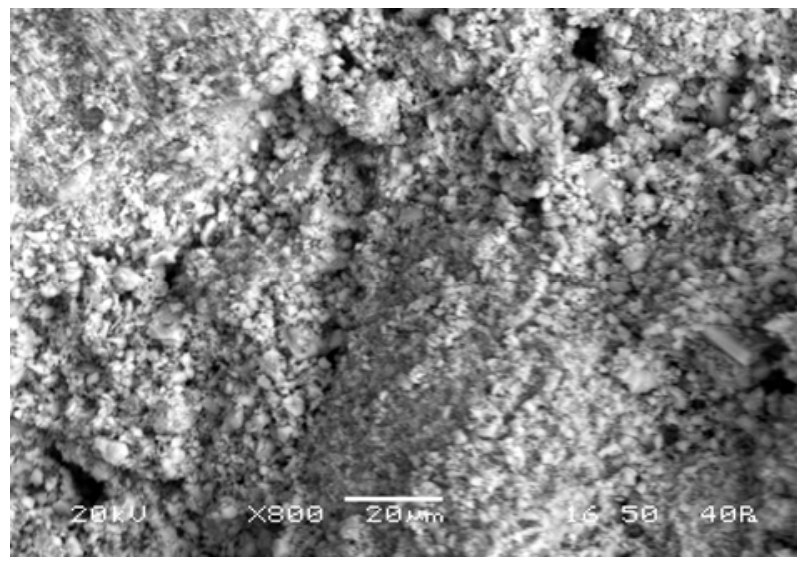

Fig. 9 Portland mortar “as supplied” grading.

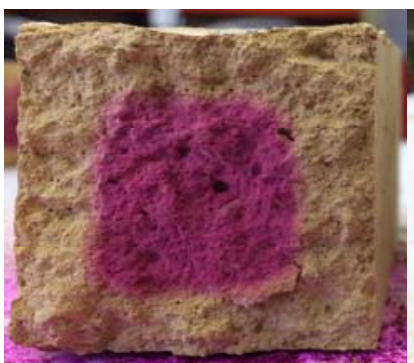

(a)

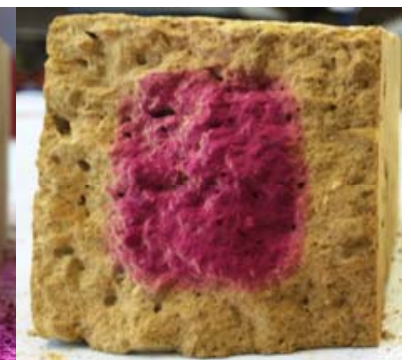

(b)
Fig. 10 Ham Hill grading: (a) "standard"; (b) "as supplied".

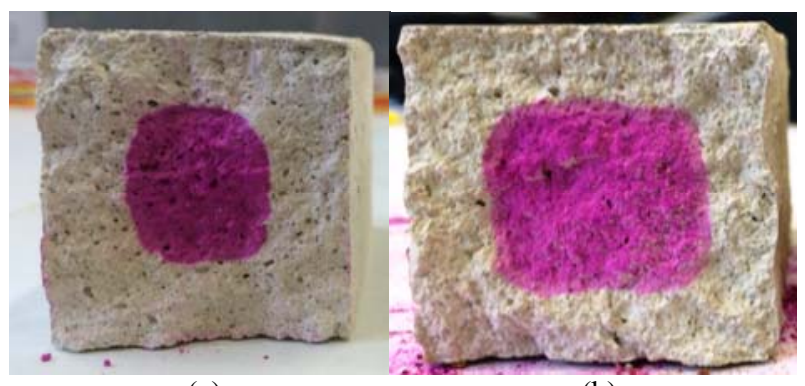

(a)

(b)

Fig. 11 Portland grading: (a) "standard"; (b) “as supplied". 


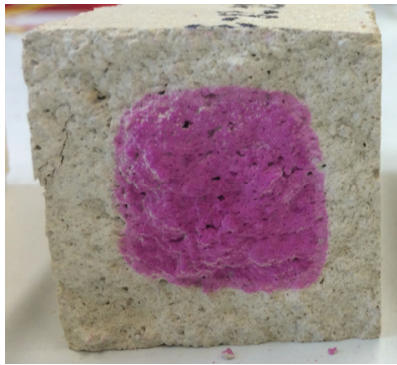

(a)

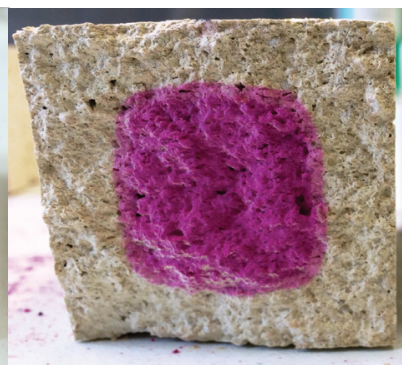

(b)

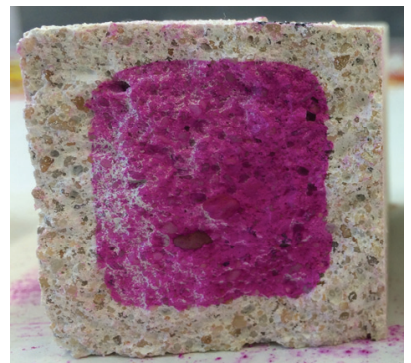

(a)

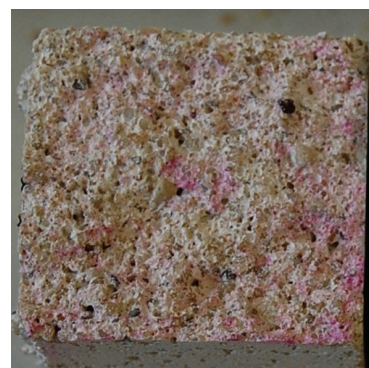

(b)

Fig. 12 Stoke Ground: (a) 28 days; (b) 360 days.

Fig. 13 Standard sand: (a) 28 days; (b) 360 days.

Table 5 Average carbonation depths.

\begin{tabular}{lll}
\hline \multirow{2}{*}{ Sample type } & \multicolumn{2}{c}{ Average depth of carbonation $(\mathrm{mm})$} \\
\cline { 2 - 3 } & "Standard" & "As supplied" \\
\hline Ham Hill & 7.6 & 7.5 \\
Portland & 10.5 & 8.0 \\
Stoke Ground & 7.5 & 7.3 \\
Standard sand & 6 & - \\
\hline
\end{tabular}

"standard" Stoke Ground sample in Fig. 5.

The Portland aggregate mortar with "as supplied" grading in Fig. 9 has fewer visible portlandite crystals, with similar size calcite crystals.

\subsection{Phenolphthalein Staining}

Since the primary setting mechanism of air lime mortar is carbonation, the depth of carbonation was measured using phenolphthalein staining. In this way, it is easy to see how much of the $\mathrm{Ca}(\mathrm{OH})_{2}$ has converted into $\mathrm{CaCO}_{3} \cdot \mathrm{Ca}(\mathrm{OH})_{2}$ is alkaline, therefore will turn pink once sprayed with phenolphthalein. $\mathrm{CaCO}_{3}$ is neutral on the $\mathrm{pH}$ scale, meaning areas of the sample that have been carbonated will remain colourless once sprayed.

Figs. 10-13 show the carbonation depths for Ham Hill, Portland, Stoke Ground and Standard sand, respectively. Table 5 summarizes the average depths.

\section{Discussions}

\subsection{Strength Results}

The compressive strength results showed what was expected as a result of the literature search; Limestone aggregate in mortar can produce higher compressive strengths than use of silicate aggregate.

There are a number of possible reasons for this that relate to aggregate type. Firstly, there is a difference in the aggregates themselves. The silicate aggregate has rounded grains, whereas the limestone aggregates are angular. This can impact the bond between the binder and aggregate, since the binder would be expected to adhere better to the angular aggregate.

Secondly, the porosity of the aggregates could be impacting the mortar strength. Limestone is more porous than silica; Consequently, it is possible that some of the binder has entered the pores of the limestone aggregate and as a result formed a stronger interface. It is also possible that due to the higher porosity of the limestone, more $\mathrm{CO}_{2}$ is able to penetrate the sample and lead to a more thorough carbonation at a given point.

Thirdly, the surface texture of the aggregate may impact the adhesion of the binder to the surface, thus resulting in stronger/weaker bond.

The "standard" graded mortars were found to be stronger than the "as supplied" mortars. This difference was greater for Stoke Ground limestone mortars, which also had the least difference in $w / b$ between the "standard" and "as supplied" mixes.

\subsection{SEM (Scanning Electron Microscopy) Analysis}

The SEM analysis has given a deeper insight into 
possible reasons for the strength differences, which is something that has been missing from literature. SEM images indicate that there are differences at the binder/aggregate interface; The development of calcite crystals is a key finding. Since binder/aggregate ratio, and particle size distribution were the same for the "standard" mixes, it is likely that aggregate type is responsible. Despite the mixes having a different water/binder ratio in order to achieve the same flow, it is not thought to influence calcite crystal growth, particularly due to the fact that Stoke Ground aggregate mortar has a significantly different $w / b$ than the other two limestones, yet it does not appear to have fewer crystals on the aggregate surface, as can be seen with the silicate aggregate.

It is currently unclear as to why the Stoke Ground aggregate mortar suffers from the highest proportion of cracks, yet it has the highest compressive strength, but could be due to the lower $w / b$ ratio that was required in order to achieve the desired flow. This is true for both the "standard" and "as supplied" mixes.

Both of the Portland mortar samples appear quite homogeneous, with the exception of one or two portlandite crystals.

\section{Conclusions and Ongoing Research}

\subsection{Conclusions}

Based on the compressive strength results and the SEM analysis, it is clear that aggregate type influences air lime mortar strength. Whilst there is currently no conclusive explanation, ongoing research is expected to clarify this.

\subsection{Ongoing Research}

Compressive strength testing and SEM analysis are also being undertaken at 14, 90, 180 and 360 days. Alongside the compressive strength testing and SEM analysis, further tests are being carried out. Thermogravimetric analysis seeks to establish differences in carbonation between the samples and at different curing periods. In this way, it will be possible to determine whether the mortars containing limestone aggregate have achieved a higher level of carbonation at a given time. Mercury intrusion porosimetry is being used to look at differences in porosity between not only the mortar samples but also the individual aggregates. To date, it has been found that key differences exist.

\section{Acknowledgments}

This work was supported by Lhoist (UK). The HIVE research building is funded by the Engineering and Physical Sciences Research Council (Grant Numbers EP/L005689/1 and EP/K040391/1).

\section{References}

[1] Mosquera, M., Benitez, D., and Perry, S. 2002. "Pore Structure in Mortars Applied on Restoration: Effect on Properties Relevant to Decay of Granite Buildings." Cement and Concrete Research 32: 1883-8.

[2] Lawrence, R. M. H. 2006. "A Study of Carbonation in Non-hydraulic Lime Mortars.” Ph.D. thesis, University of Bath.

[3] Farey, M., Holmes, S., and Livesy, M. 2003. Hydraulic Lime Mortar for Stone, Brick and Block Masonry: A Best Practice Guide. Shaftesbury: Donhead.

[4] Lanas, J., and Alvarez, J. 2002. "Masonry Repair Lime-Based Mortars: Factors Affecting the Mechanical Behaviour." Cement and Concrete Research 32: 1867-76.

[5] Arizzi, A., and Cultrone, G. 2012. "The Difference in Behaviour between Calcitic and Dolomitic Lime Mortars Set under Dry Conditions: The Relationship between Textural and Physical-Mechanical Properties." Cement and Concrete Research 42: 818-26.

[6] BIS (Department for Business Innovation \& Skills). 2010. Estimating the Amount of $\mathrm{CO}_{2}$ Emissions That the Construction Industry Can Influence. London: BIS.

[7] Lime Technology. 2013. "Limetec Mortars, Plasters and Renders.” Lime Technology. Accessed May 6, 2013. http://limetech.info/upload/documents/1160489618introto lime.pdf.

[8] Van Balen, K., and Van Gemert, D. 1994. "Modelling Lime Mortar Carbonation." Materials and Structures 27: 393-8.

[9] Skoulikidis, T. H., Charalambous, D., and Tsakona, K. 1996. "Amelioration of the Properties of Hydrated Lime for the Consolidation of the Surface or/and the Mass of Building Materials of Monuments or New Buildings or 


\section{Properties Using Scanning Electron Microscopy}

Statues and Ornaments." Presented at the 8th International Congress on Deterioration and Conservation of Stone, Berlin, Germany.

[10] British Standards Institution. 1999. BS EN 1015-11: 1999. Methods of Test for Mortar for Masonry. Determination of Flexural and Compressive Strength of Hardened Mortar. London: British Standards Institution.

[11] British Standards Institution. 1999. BS EN 1015-3: 1999. Methods of Test for Mortar for Masonry. Determination of Consistence of Fresh Mortar (by flow Table). London: British Standards Institution.

[12] Neville, A. M. 2005. Properties of Concrete. New Jersey: Pearson Prentice Hall.

[13] Lawrence, R. M. H., and Walker, P. 2008. "The Impact of the Water/Lime Ratio on the Structural Characteristics of Air Lime Mortar." In Proceedings of the 6th International Conference on Structural Analysis of Historic Construction, 885-9. 